\title{
The Mycobacterium tuberculosis sRNA F6 Modifies Expression of Essential Chaperonins, GroEL2 and GroES
}

\author{
Joanna Houghton, ${ }^{a}$ Angela Rodgers, ${ }^{\text {b }}$ Graham Rose, ${ }^{c}$ Alexandre D'Halluin, ${ }^{\text {e }}$ Terry Kipkorir, ${ }^{e}$ Declan Barker, ${ }^{\text {e Simon J. Waddell, }}{ }^{d}$ \\ (iD) Kristine B. Arnvige \\ aFaculty of Infectious Tropical Diseases, London School of Hygiene and Tropical medicine, London, United Kingdom \\ bMycobacterial Metabolism and Antibiotic Research and Host-Pathogen Interactions in Tuberculosis Laboratories, The Francis Crick Institute, London, United Kingdom \\ cNorth Thames Genomic Laboratory Hub, Great Ormond Street Hospital for Children, London, United Kingdom \\ ¿Global Health and Infection, Brighton and Sussex Medical School, University of Sussex, Brighton, United Kingdom \\ eStructural and Molecular Biology, University College London, London, United Kingdom
}

ABSTRACT Almost 140 years after the identification of Mycobacterium tuberculosis as the etiological agent of tuberculosis, important aspects of its biology remain poorly described. Little is known about the role of posttranscriptional control of gene expression and RNA biology, including the role of most of the small RNAs (sRNAs) identified to date. We have carried out a detailed investigation of the M. tuberculosis sRNA F6 and shown it to be dependent on SigF for expression and significantly induced in starvation conditions in vitro and in a mouse model of infection. Further exploration of F6 using an in vitro starvation model of infection indicates that F6 affects the expression of the essential chaperonins GroEL2 and GroES. Our results point toward a role for F6 during periods of low metabolic activity typically associated with long-term survival of $M$. tuberculosis in human granulomas.

IMPORTANCE Control of gene expression via small regulatory RNAs (sRNAs) is poorly understood in one of the most successful pathogens, Mycobacterium tuberculosis. Here, we present an in-depth characterization of the sRNA F6, including its expression in different infection models and the differential gene expression observed upon deletion of the sRNA. Our results demonstrate that deletion of F6 leads to dysregulation of the two essential chaperonins GroEL2 and GroES and, moreover, indicate a role for F6 in the long-term survival and persistence of M. tuberculosis in the human host.

KEYWORDS Mycobacterium tuberculosis, small RNA, nutrient starvation, Wayne model chaperonins, infection, hypoxia, infection models, microarrays, persistence, small regulatory RNA

ne hundred years after the launch of the Bacillus Calmette-Guerin (BCG) vaccine against tuberculosis (TB), this disease still claims more than 3,000 lives on a daily basis, and its etiological agent, Mycobacterium tuberculosis, remains one of the most prominent pathogens in human history. Notoriously difficult to work with and very different from both Gram-positive and Gram-negative model organisms, many aspects of its basic biology, including RNA biology and posttranscriptional control of gene expression, remain unclear. However, the study of regulatory RNA in M. tuberculosis is gaining momentum, aided by next-generation sequencing (NGS) applications, which have provided significant insights into the abundance and dynamics of noncoding RNA over a range of growth conditions, the location of transcription start sites on a global scale, and translated versus untranslated transcripts (1-5). Still, much remains to be uncovered about the posttranscriptional control exerted by regulatory RNA in M. tuberculosis, in particular what role these molecules play in the pathogenesis and persistence of $M$. tuberculosis. A multitude of $M$. tuberculosis small regulatory RNAs (sRNAs) have been
Citation Houghton J, Rodgers A, Rose G, D'Halluin A, Kipkorir T, Barker D, Waddell SJ, Arnvig KB. 2021. The Mycobacterium tuberculosis sRNA F6 modifies expression of essential chaperonins, GroEL2 and GroES. Microbiol Spectr 9:e01095-21. https://doi.org/ 10.1128/Spectrum.01095-21.

Editor Amanda G. Oglesby, University of Maryland School of Pharmacy

Copyright $\odot 2021$ Houghton et al. This is an open-access article distributed under the terms of the Creative Commons Attribution 4.0 International license.

Address correspondence to Kristine B. Arnvig, k.arnvig@ucl.ac.uk.

Received 29 July 2021

Accepted 25 August 2021

Published 22 September 2021 
identified in the last decade, but only few have been investigated, and even fewer have been linked to $M$. tuberculosis persistence and TB latency $(1,3,4,6-13)$.

F6 (ncRv10243) was one of the first M. tuberculosis sRNAs to be identified by cDNA cloning (6). F6 is conserved in a wide range of mycobacteria with the $5^{\prime}$ end showing the highest degree of conservation $(6,7)$. The location of $F 6$ between the convergent fadA2 (encoding an acetyl coenzyme A [acetyl-CoA] transferase) and fadE5 (encoding an acyl-CoA hydrogenase) is also highly conserved, suggesting a potential role for F6 in the regulation of lipid metabolism, which is critical for intracellular survival (14). Initial analysis, combining Northern blotting with $5^{\prime}$ and 3' rapid amplification of CDNA ends (RACE), revealed that F6 is expressed as a 102-nucleotide transcript, which is $3^{\prime}$ processed to the dominant transcript of 58 nucleotides (6). F6 is upregulated in the stationary phase, during oxidative stress, and by low $\mathrm{pH}$, while overexpression from a multicopy-number plasmid leads to a slowgrowth phenotype on solid media (6). The F6 promoter contains a typical SigF promoter motif and exhibits the highest SigF occupancy according to chromatin immunoprecipitation with microarray technology (ChIP-chip) analysis $(6,15)$. SigF is a nonessential sigma factor, conserved in most mycobacteria; its expression is induced by several stresses, including anaerobiosis, nutrient starvation, oxidative stress, and cold shock, while heat shock downregulates its expression, and deletion leads to attenuation in mice (16-19).

In the current study, we have investigated M. tuberculosis F6 expression under different growth conditions and found that this SRNA is significantly upregulated in a nutrient starvation model of persistence and in mouse lungs. We have generated an F6 deletion strain of $M$. tuberculosis and assessed the fitness of the $\Delta f 6 M$. tuberculosis strain in vitro and in vivo. We found that inactivation of F6 leads to impaired recovery from a Wayne model of hypoxia. Moreover, using microarray analysis and reverse transcription-quantitative PCR (qRT-PCR), we have found that expression of the essential chaperonins encoded by groEL2/rv0440 was significantly upregulated upon deletion of F6. Our results suggest that although F6 is highly upregulated in M. tuberculosis during the early stages of infection, it is likely to play a more prominent role during later stages of infection.

\section{RESULTS}

Expression of F6/SfdS is upregulated by starvation and during infection. SigF is the predicted main regulator of F6 expression, and this was validated by Northern blotting, which demonstrated a complete absence of F6 in a $\Delta$ sigF strain (Fig. S1). We will henceforth refer to F6 as SfdS, for SigF-dependent sRNA. SigF is highly expressed during infection and in persister in vitro models such as nutrient starvation obtained by static incubation in phosphate-buffered saline (PBS) (16). We subjected M. tuberculosis H37Rv to starvation by washing and resuspending log-phase cultures in PBS for 24 and $96 \mathrm{~h}$ before isolating total RNA. SfdS expression was measured by qRT-PCR (normalized to $16 \mathrm{~S}$ rRNA) and compared to log-phase levels.

The results demonstrate that SfdS expression increased dramatically (19-fold) within $24 \mathrm{~h}$ of starvation, with levels of SfdS remaining elevated for at least another $72 \mathrm{~h}$ (Fig. 1).

To investigate SfdS expression in a mouse model of infection, BALB/c mice were infected with $\sim 100 \mathrm{CFU}$ of H37Rv via aerosol route and left for 3 weeks before culling and isolation of M. tuberculosis total RNA from lung tissue. We found that SfdS expression was robustly upregulated ( $\sim 6$-fold) compared to log-phase levels but not to the same extent as that during in vitro starvation (Fig. 1).

Together, these results demonstrate that the expression of SfdS is dynamic and that it may play a role during infection, more specifically in nutrient-poor environments.

Deletion of SfdS does not impair M. tuberculosis standard growth in vitro. To identify a potential regulatory role of SfdS with minimal impact on the two flanking fad genes, we generated an unmarked SfdS deletion strain using site-directed mutagenesis and allelic exchange. Candidates were screened by PCR amplification and 


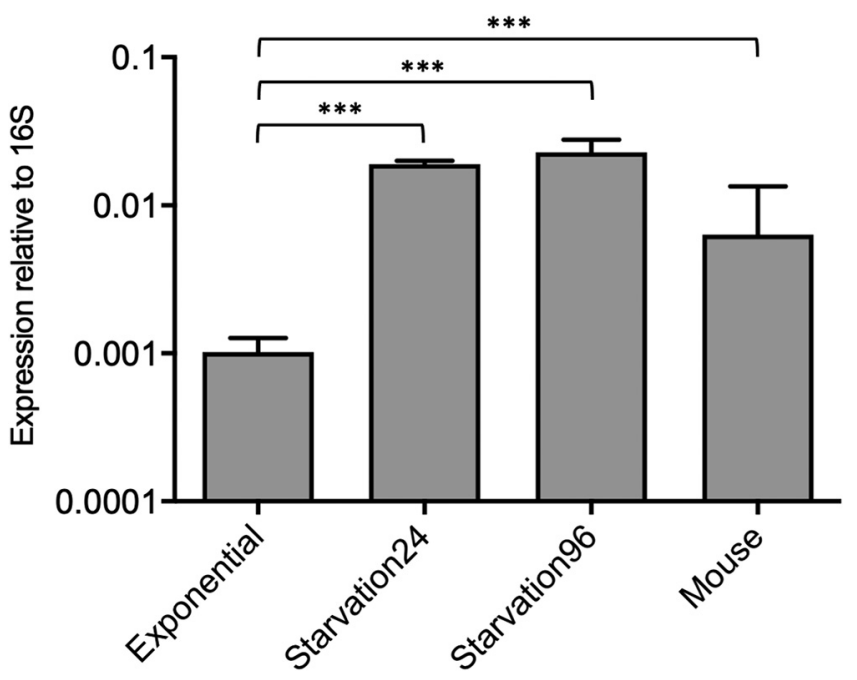

FIG 1 Expression of SfdS in M. tuberculosis. SfdS expression levels were measured in exponentially growing cultures, after 24 and 96 h in PBS (Starvation24 and Starvation96) and in mouse lungs after 3 weeks of infection using quantitative RT-PCT (qRT-PCR). Expression levels were normalized to 16S rRNA, and the data represent the mean and standard deviation of three biological replicates for each condition. ${ }^{* *}, P$ value of $<0.05$ with significance tested using one-way analysis of variance (ANOVA).

sequencing of the PCR product, which confirmed the deletion of the sRNA from the $M$. tuberculosis genome (not shown).

To further verify the deletion and to determine if there were secondary mutations that might affect subsequent phenotypic analysis, wild-type and $\Delta s f d S$ strains were sequenced. Alignment of the genomic sequence of $\Delta s f d S$ with H37Rv identified two single-nucleotide polymorphisms (SNPs) in addition to the Xba I site deliberately introduced; one was C2864730T, resulting in an R102W substitution in Rv2541 (hypothetical protein), while the other was C4178146T in the $5^{\prime}$ untranslated region (UTR) of Rv3729 (potential transferase). To ensure that these SNPs did not influence the phenotype of the SfdS deletion strain, we constructed a complemented strain, in which expression of SfdS was driven by its native promoter from a single-copy plasmid integrated on the chromosome. Growth of wild-type H37Rv, $\Delta s f d S$, and the complemented strain was monitored over a period of 2 weeks in $7 \mathrm{H} 9$ roller bottle cultures. Under these conditions, there was no significant difference between the three strains (Fig. 2).

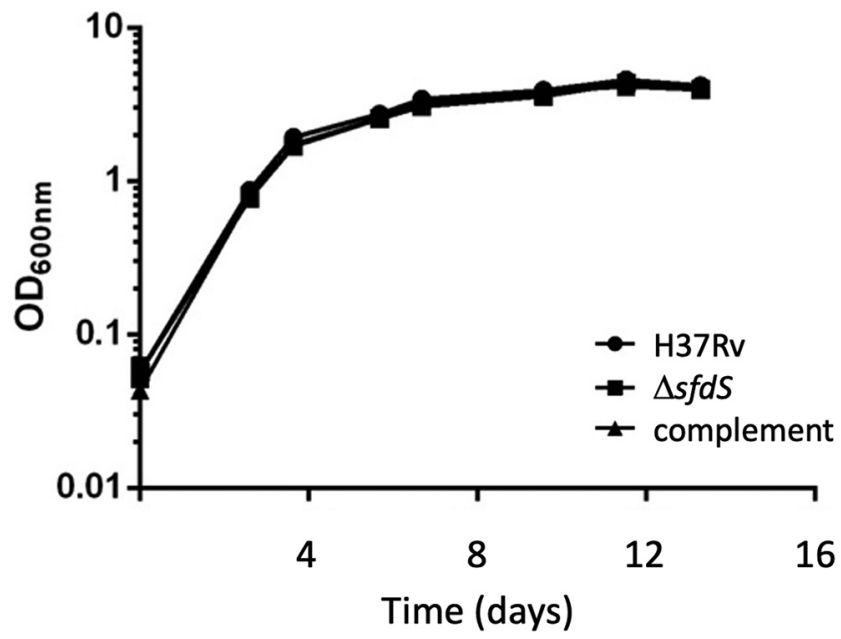

FIG 2 In vitro growth of $\Delta s f d S$. Growth curves of wild-type M. tuberculosis H37Rv, $\Delta s f d S$, and its complement in $7 \mathrm{H} 9$ in roller bottles. The results represent the average and standard deviation of three biological replicates. 


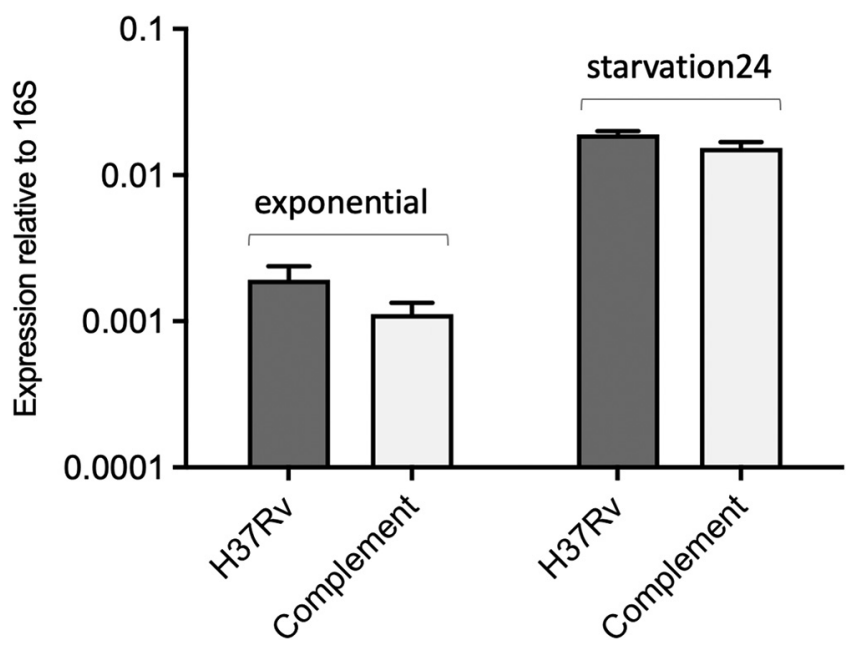

FIG 3 Expression of SfdS in wild-type H37Rv and complemented strain. RNA was isolated from logphase and after $24 \mathrm{~h}$ starvation and analyzed by qRT-PCR. Data represent the mean and standard deviation of three biological replicates for each strain. Differences between wild-type and complement are not significant according to unpaired $t$ test.

To validate deletion and complementation, expression of SfdS was measured in wild-type H37Rv, $\Delta s f d S$, and the complemented strain during exponential growth and after $24 \mathrm{~h}$ of PBS starvation, using qRT-PCR. The results demonstrated that there was no detectable expression of SfdS in the deletion strain (Fig. S2) and that there was a small, nonsignificant difference between SfdS expression in wild-type and complemented strains in both growth conditions (unpaired $t$ test, $P<0.05$, Fig. 3).

Fitness of the $\Delta s f d S$ strain in macrophages and mouse models of infection. As we had observed a robust upregulation of SfdS during infection, we wanted to compare the fitness of wild-type M. tuberculosis H37Rv, $\Delta s f d S$, and the complemented strain during infection. The three strains were used to infect naive and interferon gamma (IFN- $\gamma$ ) preactivated murine bone marrow-derived macrophages (BMDMs) at a multiplicity of infection (MOI) of 0.1:1 (bacteria/macrophages). The infection was allowed to continue for 7 days with five time points. At each time point, the macrophages were lysed and plated for CFU. For both naive and activated macrophages, we observed no significant differences between the three strains, suggesting that under these conditions, deletion of SfdS does not result in attenuation (Fig. 4A).

A macrophage model of infection does not entirely replicate the conditions encountered by the bacteria in more complex animal models of infection. To assess the role of SfdS in pathogenesis in a more representative infection model, wild-type $M$. tuberculosis H37Rv, $\Delta s f d S$, and the complemented strain were compared in a mouse model of infection. BALB/C mice were infected with approximately $100 \mathrm{CFU}$ via the aerosol route, and the infection was followed for 143 days. On days $0,30,78,100$, and 143 , lungs and spleens were harvested for bacterial enumeration. Again, no significant difference was observed in CFU between the wild-type M. tuberculosis H37Rv and $\Delta s f d S$ at any of the time points (Fig. 4B).

Recovery from Wayne model hypoxia is impaired in $\Delta s f d S$. One of the hallmarks of human TB is the formation of granulomas, which are absent in a standard mouse model. Granulomas are characterized by limited nutrient and oxygen availability, among other things (20). A widely used in vitro model of hypoxia is the Wayne model, in which the available oxygen is gradually limited in sealed cultures of $M$. tuberculosis (21). When oxygen concentrations decrease to the microaerobic level ( $1 \%$ oxygen saturation), the cells enter a state of nonreplicating persistence, NRP-1, followed by NRP-2 when oxygen saturation reaches $0.06 \%$. SigF is highly induced during anaerobiosis, suggesting that its regulon, including SfdS, may play a role in low-oxygen conditions (19). We therefore decided to evaluate the fitness of the $\Delta s f d S$ strain using the Wayne 
A

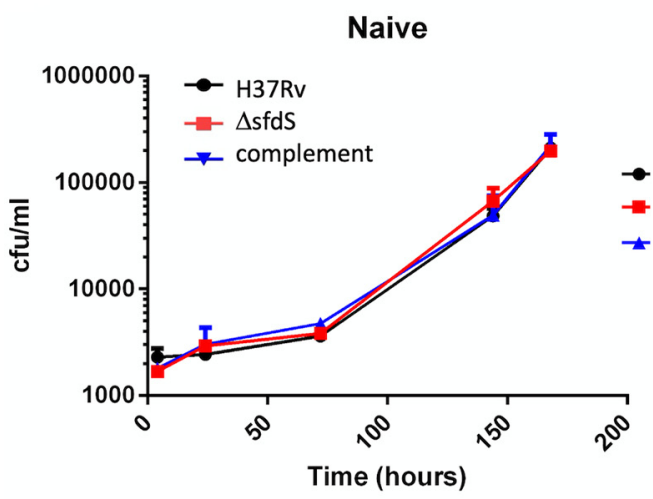

\section{Macrophage infections}

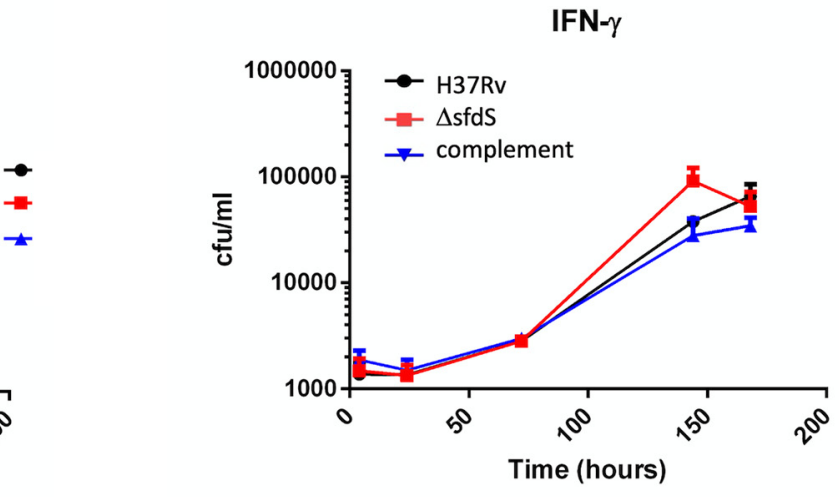

B

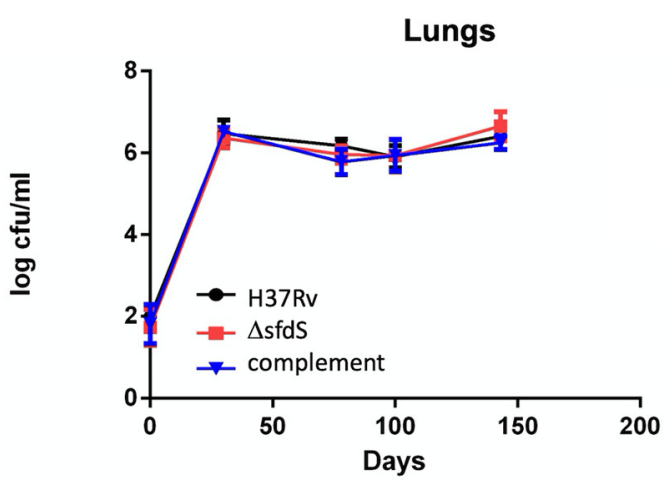

\section{Mouse infections}

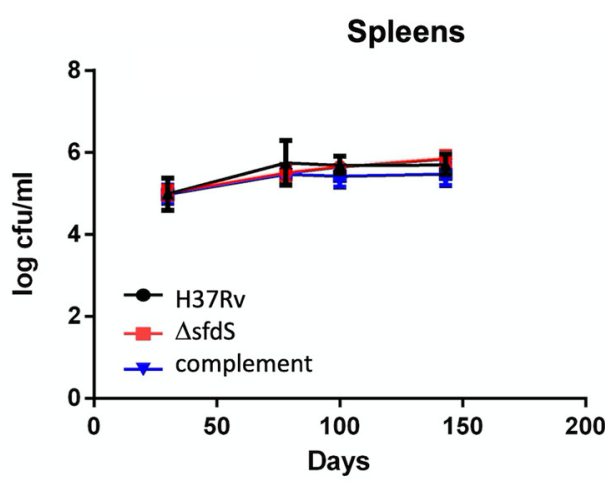

FIG 4 Survival of the $\Delta s f d S$ in models of infection. Panel A shows survival of wild-type M. tuberculosis H37Rv, $\Delta$ sfdS, and complementing strains in a macrophage model of infection with naive (left) and IFN- $\gamma$ activated (right) BMDM. Data represent the mean and standard deviation of triplicate infections. Statistical significance was tested with one-way ANOVA, $P<0.05$. Panel B shows survival of the three strains within the lungs and spleens of BALB/c mice. Data represent the averages and standard deviations from 5 mice per time point. Statistical significance was tested with two-way ANOVA.

model. To measure potential differences in respiration rate, the depletion of oxygen for each strain was monitored by a methylene blue indicator tube set up for each strain at the start of incubation. After 14 days of incubation, at NRP-2, cultures were plated onto $7 \mathrm{H} 11$ agar for determination of CFU. In addition, NRP-2 cells were assessed for their ability to be resuscitated by dilution into fresh 7H9 media and monitoring of growth (by optical density at $600 \mathrm{~nm}\left[\mathrm{OD}_{600}\right]$ ). There was no significant difference in survival (CFU) between wild-type H37Rv, $\Delta s f d S$, and the complemented M. tuberculosis strain at day 14 incubation in the Wayne model (Fig. 5A). Conversely, the regrowth of NRP-2 cells transferred to fresh media indicated that the $\Delta s f d S$ strain was impaired for recovery/resuscitation following incubation in the Wayne model, while the complemented strain displayed an intermediate phenotype (Fig. 5B). These results suggest that $\mathrm{SfdS}$ may play a role in the resuscitation of nonreplicating $M$. tuberculosis, which is associated with the reactivation of latent infection.

Deletion of SfdS leads to upregulation of essential chaperonins. To establish a potential regulatory role for SfdS, we employed nutrient starvation, as this was the most potent inducer of the sRNA in our hands. RNA was isolated from triplicate cultures of wild-type H37Rv and $\Delta$ sfdS starved for $24 \mathrm{~h}$ in PBS and analyzed using Agilent microarrays.

Strikingly, only a single gene, groEL2/rv0440, was significantly differentially expressed, with a 3-fold upregulation in the $\Delta s f d S$ strain compared to that in wild-type H37Rv (Fig. 6). However, with a less-stringent analysis, i.e., removing the multiple testing correction but maintaining the cutoff at a 2-fold change, we observed nine differentially expressed genes, 

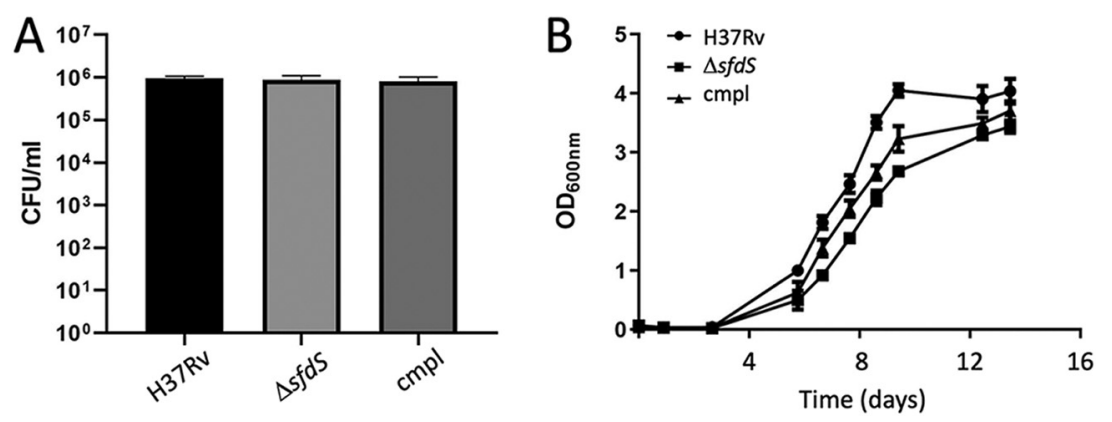

FIG 5 Recovery from NRP-2 is impaired in $\Delta s f d S$. Wild-type M. tuberculosis H37Rv, $\Delta s f d S$, and complement $(\mathrm{cmpl})$ strains were grown in the Wayne model until NRP-2 was reached. Cultures were diluted and plated for CFU counts. (A) Number of viable bacteria after incubation in the Wayne model. (B) All cultures were subsequently adjusted to an $\mathrm{OD}_{600}$ of 0.05 in fresh media, and growth was measured over 14 days. All data represent the averages and standard deviation of three biological replicates. Paired $t$ test between wild-type and $\Delta s f d S$ as well as between $\Delta s f d S$ and complement indicated a significant difference $(P<0.05)$ in postrecovery growth.

of which five (dnaK, groEL2, rv0990c, rv2012, and rv3224) are associated with heat shock in M. tuberculosis (Fig. S3) (22).

In an effort to identify potential, direct mRNA targets of SfdS, we used the TargetRNA2 webserver with default settings and full-length SfdS against the nine differentially expressed genes (23). However, no direct targets could be predicted in this way. Instead, we assumed that the observed changes represent downstream effects and hypothesized that the differentially expressed genes had a common regulator. Four of the nine genes (dnaK, groEL2, rv0990c, and rv3224) are specifically associated with HrCA, which directly regulates transcription of groEL2, the groES-groEL1 and rv0991c-rv0990c operons, and its own expression, i.e., the hrcA-dnaJ2 operon $(22,24)$. To ensure that expression of groEL2 was restored to wild-type levels in the complemented strain, we performed qRT-PCR on RNA extracted from three biological replicates of wild-type H37Rv, $\Delta s f d S$, and the complemented strain. The results confirmed that groEL2 was significantly upregulated in $\Delta s f d S$ upon starvation and that the phenotype could be complemented by providing a copy of the sfdS gene in trans (Fig. 7). To probe if the observed changes in groEL2 expression could be associated with transcriptional control by HrcA, we also measured the expression of the first gene in each of the HrcA-controlled operons, i.e., groES, rv0991c, and hrcA, in the three M. tuberculosis strains. Similar to the expression of groEL2, that of groES was significantly upregulated in $\Delta s f d S$ and restored in the complemented strain; $r$ 0991c and $h r c A$ displayed similar trends, but the changes were not statistically significant, and therefore the role of HrcA remains inconclusive (Fig. 7).

\section{DISCUSSION}

The M. tuberculosis SigF-controlled sRNA SfdS (F6) is well expressed in the exponential phase but upregulated by a variety of stresses (6).

In this study, we have shown that SfdS is also highly upregulated after 3 weeks of murine infection and, to an even higher extent, in the PBS starvation model at 24 and $96 \mathrm{~h}$. This suggests that SfdS plays a role in the early stages of pathogenesis but possibly a more significant one for survival in nutrient-deficient environments, such as those encountered in human granulomas in later stages of infection. M. tuberculosis may resuscitate after periods of low metabolic activity $(21,25,26)$, and using the Wayne model of nonreplicating persistence, we found that recovery of $\Delta s f d S$ from NRP-2 was impaired compared to wild-type and complemented strains, although initial survival was unchanged. The fact that the complemented strain displayed an intermediate phenotype may be due to cis-regulatory effects associated with the deletion, the different genomic location of the complementing $\mathrm{SfdS}$, or the point mutations identified with the whole-genome sequencing (WGS). 


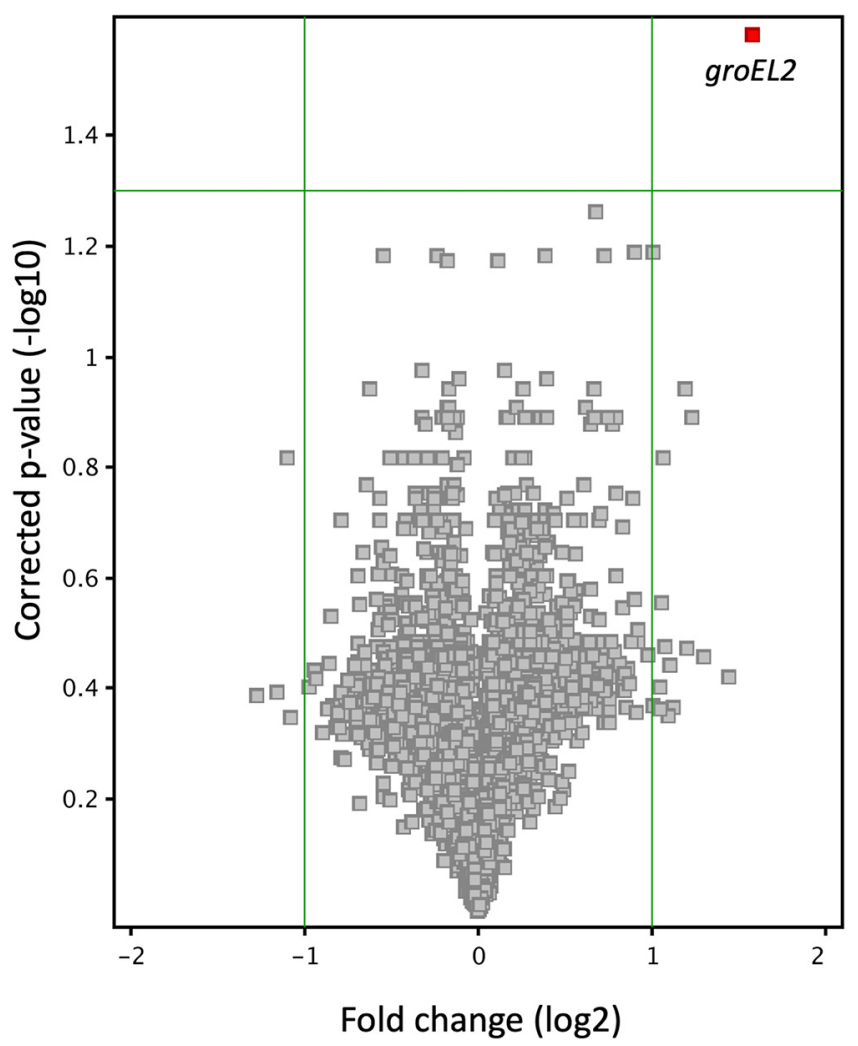

FIG 6 Volcano plot of $\Delta$ sfdS versus H37Rv upon starvation in PBS. The plot shows groEL2 as the only gene whose expression level was significantly changed ( $\geq 2$-fold differentially expressed) between $\Delta$ sfdS and H37Rv.

Our results indicate that starvation for $24 \mathrm{~h}$ leads to the upregulation of HrcA-controlled chaperonins groES and groEL2 in the $\triangle$ sfdS strain compared to the expression in the wild-type and complemented $M$. tuberculosis strains, while the expression of the remaining HrcA-regulated operons (rv0991c-0990c and hrcA-dnaJ2) was not significantly changed. However, regulation of HrcA expression and activity is highly complex and the lack of a significant change of $h r c A$ mRNA levels in the $\Delta$ sfdS strain does not exclude a role for this regulator in the observed response.

Prediction of potential mRNA targets of SfdS using the TargetRNA2 webserver (23) did not result in any direct targets, and we conclude that the observed changes likely represent downstream effects from other, as yet unidentified targets.

Nevertheless, the fact that SfdS appears to repress chaperonin expression does offer a potential explanation for the previously observed slow growth associated with SfdS overexpression (6), namely, an untimely repression of these essential chaperonins, which could lead to reduced protein synthesis. This also suggests that the overexpression strain may be hypersensitive to heat shock.

RNA is a ubiquitous temperature-sensing molecule, and several heat shock response mechanisms employ RNA, including the eukaryotic heat shock transcription factor 1 (HSF1), Escherichia coli rpoH, and Deinococcus radiodurans sRNA DnrH, the latter of which activates the expression of Hsp20 (27-29). Further analysis using pulsed rather than constitutive overexpression or deletion may provide more clues on the link between SfdS and heat shock.

Infection of BMDMs for 7 days with H37Rv, $\Delta s f d s$, and complement revealed no attenuation of the deletion strain. Similarly, we observed no difference in the CFU recovered from the lungs and spleens of BALB/C mice after 143 days of infection. As $\mathrm{SfdS}$ expression is not only directed by SigF but also dominant in terms of promoter 


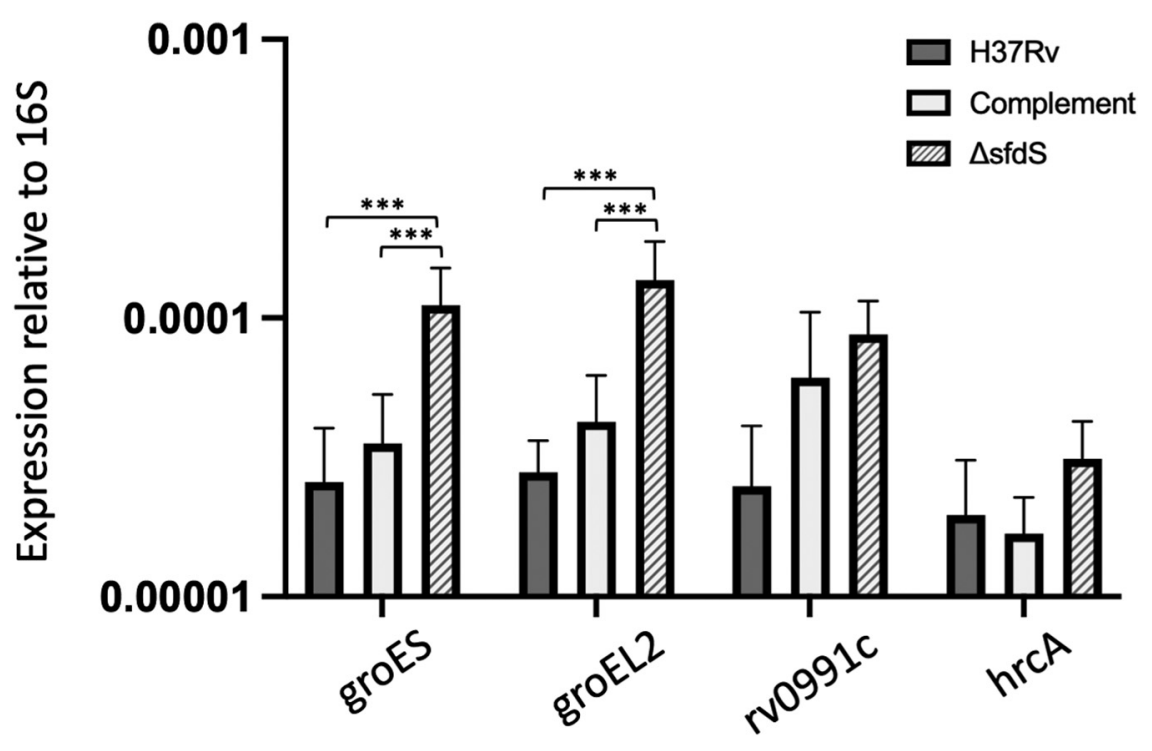

FIG 7 Expression of HrcA-regulated genes in M. tuberculosis strains. qRT-PCR was performed on total RNA from $M$. tuberculosis H37Rv (wild-type), $\Delta$ sfdS mutant, and its complement. Each bar shows the expression level of the indicated genes normalized to 16S rRNA. All data represent the mean and standard deviation of three biological replicates for each strain. ${ }^{* *}$, $P$ value of $<0.05$ with significance tested using one-way ANOVA.

occupancy, one might expect some parallels between infection with $\Delta s f d S$ and $\Delta s i g F$. Indeed, a lack of attenuation has been observed in a study of a $\Delta s i g F$ strain in human monocyte derived macrophages, while late stages of infection and time-to-death experiments result in attenuation of a CDC1551 $\Delta$ sigF strain $(18,30)$. Given that starvation is a potent inducer of SigF/SfdS expression, it seems possible that a more long-term infection and/or the ability to form granulomas is required to observe strong phenotypes associated with the deletion of SfdS. Alternatively, a higher MOI might change the course of infection. Moreover, the mouse model of infection does not replicate all aspects of an $M$. tuberculosis infection in humans, one key difference being the lack of granuloma formation in a standard mouse model such as BALB/C (31). Granulomas provide a unique niche for $M$. tuberculosis in which oxygen and nutrients are limited, the latter being the strongest inducer of SfdS expression in our study. A different animal model, e.g., C3HeB/FeJ mice, which develop necrotic lung granulomas, may provide more answers (32). Our results point toward a role for SfdS during periods of low metabolic activity similar to those of cold shock and may be associated with nutrient starvation conditions, such as those found in human granulomas in later stages of infection, and it remains a possibility that SfdS plays a role in surviving and resuscitating from this environment.

\section{MATERIALS AND METHODS}

Bacterial strains and plasmids. Escherichia coli $\mathrm{DH} 5 \alpha$ was used for plasmid construction and grown in LB agar or broth using kanamycin at $50 \mu \mathrm{g} / \mathrm{ml}$ and X-Gal where necessary at $200 \mu \mathrm{g} / \mathrm{ml}$. Mycobacterium tuberculosis $\mathrm{H} 37 \mathrm{Rv}$ was grown on Middlebrook $7 \mathrm{H} 11$ agar plus $10 \%$ oleic acid-albumin-dextrose-catalase (OADC) (Becton, Dickinson). Liquid cultures were grown in standard Middlebrook 7H9 medium supplemented with $0.5 \%$ glycerol, $10 \%$ Middlebrook ADC (Becton, Dickinson), and $0.05 \%$ Tween 80 at $37^{\circ} \mathrm{C}$ in a roller bottle (Nalgene) rolling at $2 \mathrm{rpm}$ or 50-ml falcon tubes (Corning) in an SB3 tube rotator (Stewart) at $28 \mathrm{rpm}$. Kanamycin was added where required at $25 \mu \mathrm{g} / \mathrm{ml}$ and X-Gal where required at $50 \mu \mathrm{g} / \mathrm{ml}$. All plasmids and oligonucleotides used in this study are listed in Tables S1 and S2.

The Wayne model. Cultures were grown to exponential phase $\left(\mathrm{OD}_{600}=0.6\right.$ to 0.8$)$ and subsequently diluted to an $\mathrm{OD}_{600}$ of 0.005 in $7 \mathrm{H} 9$ in triplicate in Wayne tubes, each containing a sterile stirring bar. Cultures were incubated at $37^{\circ} \mathrm{C}$ on a stirring platform, and $\mathrm{OD}$ was monitored over time until cultures reached NRP-2. Cultures were then either diluted into $7 \mathrm{H} 9, \mathrm{OD}_{600} 0.05$, and $\mathrm{OD}$ monitored or serially diluted onto $7 \mathrm{H} 11$ agar and CFU enumerated.

Construction of $\mathbf{F 6 / S f d S ~ d e l e t i o n ~ a n d ~ c o m p l e m e n t e d ~ s t r a i n s . ~ A l l e l i c ~ r e p l a c e m e n t ~ t e c h n i q u e s ~ w e r e ~}$ used to generate an M. tuberculosis knockout mutant as per published protocol (33). Briefly, approximately $1.5 \mathrm{~kb}$ of the flanking region from either side of F6/SfdS (5' flank coordinates 292520 to 293605 
and 3' flank 293709 to 294600) was cloned into the suicide vector pBackbone (34). The sRNA was replaced by an Xba I site using site-directed mutagenesis to produce the targeting plasmid pJHP04. Electrocompetent H37Rv was transformed with pJHP04, and subsequent single crossovers (SCOs) and double crossovers (DCOs) were selected (see supplemental material for details).

To complement the $\Delta f 6 / s f d S$ mutant, electrocompetent cells were prepared and transformed with an integrating plasmid (pJHP06) containing the cloned region 293428 to 293876 from M. tuberculosis H37Rv and supplemented with pBSInt, providing the phage integrase (35). Complementation was confirmed using qRT-PCR.

Mapping genome data and variant calling. To determine the genome sequence of the deletion strain, WGS was performed as described elsewhere using the Illumina HiSeq platform (2). Sequencing reads for the $\Delta f 6 / s f d S$ strain were mapped against the $M$. tuberculosis $H 37 R v$ reference genome (AL123456) using Burrows-Wheeler aligner (BWA) (36), and variants were called with SAMtools (37). Variant filtering was performed by inclusion of only those variants with a minimum mapping quality of 10 and maximum read depth of 400 . Finally, heterozygous calls or those found in repetitive or mobile elements (genes annotated as PE/PPE/insertions/phages) were removed.

Preparation of starved cultures. M. tuberculosis H37Rv, $\Delta f 6 / s f d S$, and complemented strain were grown in Middlebrook $7 \mathrm{H} 9$ supplemented with $0.4 \%$ glycerol, $0.085 \% \mathrm{NaCl}, 0.5 \%$ bovine serum albumin (BSA), and $0.05 \%$ tyloxapol in roller bottle culture $\left(2 \mathrm{rpm}\right.$ at $\left.37^{\circ} \mathrm{C}\right)$. Exponentially growing bacteria were pelleted and washed 3 times with PBS supplemented with $0.025 \%$ tyloxapol and finally resuspended in triplicate in PBS with $0.025 \%$ tyloxapol to an equivalent starting volume and incubated statically for 24 or $96 \mathrm{~h}$.

RNA isolation. RNA isolation from in vitro cultures was done as described previously (6). Briefly, we harvested cultures with rapid cooling by adding ice directly to the culture and subsequent centrifugation at 10,000 rpm for 10 min. RNA was isolated from the pellet using the FastRNA pro blue kit from MP Biomedicals following the manufacturer's instructions.

To isolate RNA from bacteria grown in mice, lung homogenates were spun at 13,000 rpm for 5 min to collect the bacteria. These were resuspended in $1 \mathrm{ml} \mathrm{TRIzol}$ (Invitrogen) with $150-\mu \mathrm{m}$ glass beads, and the samples were disrupted in a FastPrep (MP Biomedicals) at a setting of 6.0 for $40 \mathrm{~s}$. The RNA was extracted according to the manufacturer's guidelines. RNA concentration was measured by Nanodrop (Thermo Scientific), and RNA integrity was measured by the 2100 Bioanalyzer using a nano chip (Agilent Technologies).

Northern blotting. Ten micrograms each of H37Rv and $\Delta$ sigF total RNA was separated on a $10 \%$ denaturing acrylamide gel and transferred onto Brightstar-Plus nylon membrane (Ambion) by electroblotting. RNA was UV crosslinked to the membrane and stained with $0.3 \mathrm{M}$ sodium acetate/0.03\% methylene blue to verify transfer. 32P-labeled riboprobes were synthesized using the mirVana probe construction kit (Ambion) and 32P-UTP $(800 \mathrm{mCi} / \mathrm{mmol}$, PerkinElmer) with the template oligonucleotide listed in Table S2 and hybridized to the membranes overnight in UltraHyb (Invitrogen).

Quantitative RT-PCR. Total RNA was treated with Turbo DNase (Ambion) until DNA free. cDNA was synthesized using Superscript III (Invitrogen) and random hexamers. Primers were designed using the Applied Biosystems software Primer Express, and sequences are listed in Table S1. Each 20- $\mu$ I qRT-PCR contained 16SYBRgreen (Applied Biosystems), $900 \mathrm{nM}$ each primer, and $5 \mu$ l of template cDNA. Absolute quantitation was carried out, and all genes were normalized to 16S rRNA expression.

Transcriptional profiling. Whole-genome $M$. tuberculosis microarray slides were purchased from Agilent Technologies through the Bacterial Microarray Group at St. George's (B $\mu \mathrm{G} @ S)$, University of London. For cDNA synthesis, $2 \mu \mathrm{g}$ wild-type H37Rv and $\Delta s f d S$ knockout isolated from 24-h starved cultures was used. The cDNA was labeled individually with both Cy-3 and Cy-5 dyes (GE Healthcare) using Superscript III reverse transcriptase (Invitrogen). Dye swaps were performed, and the cDNA was hybridized to an $8 \mathrm{Chamber}$ Agilent slide at $65^{\circ} \mathrm{C}$ for $16 \mathrm{~h}$ before slide was washed with Oligo aCGH wash buffer 1 (Agilent) for $5 \mathrm{~min}$ at room temperature and Oligo aCGH wash buffer 2 (Agilent) for $1 \mathrm{~min}$ at $37^{\circ} \mathrm{C}$. Slides were stabilized using Agilent stabilization and drying solution according to the manufacturer's instructions.

Slides were scanned at $5 \mu \mathrm{m}$ using an Agilent Technologies microarray scanner at B $\mu \mathrm{G} @ \mathrm{~S}$. Txt files created by the Agilent scanner were analyzed using Genespring 14.5 filtering on flags and expression. $t$ test against zero was performed using a $P$ value of $<0.05$ with Benjamini-Hochberg multiple testing correction and 2-fold cutoff. Array design is available in ArrayExpress (accession no. A-BUGS-41). Microarray data have been deposited into ArrayExpress (accession number E-MTAB-9327).

F6 target prediction. Prediction of potential F6 sRNA targets was performed using the TargetRNA2 webserver (http://cs.wellesley.edu/ btjaden/TargetRNA2/index.html) with differentially expressed genes and default parameters (23).

Macrophage infection. Bone marrow-derived macrophages (BMDMs) were generated from 6- to 8week-old BALB/c mice in RPMI 1640 (Gibco) containing 10\% fetal calf serum, $20 \mu \mathrm{M}$ L-glutamine, $1 \mathrm{mM}$ sodium pyruvate, $10 \mu \mathrm{M}$ HEPES, and $50 \mathrm{nM} \beta$-mercaptoethanol. The cells were then grown and differentiated in complete RPMI 1640 supplemented with $20 \%$ L929 cell supernatant for 6 days at $37^{\circ} \mathrm{C}$ in $5 \%$ $\mathrm{CO}_{2}$. The differentiated cells were seeded at a density of $2 \times 10^{5}$ cells/well in $1 \mathrm{ml}$ complete RPMI 1640 supplemented with 5\% L929 cell supernatant and incubated overnight prior to infection. M. tuberculosis strains for infection were grown to an $\mathrm{OD}_{600}$ of 0.5 to 0.8 , and inocula were prepared by washing and resuspending the cultures in PBS to produce a single cell suspension. This was used to infect BMDMs at a multiplicity of infection (MOI) of 0.1:1. After $4 \mathrm{~h}$, the cells were washed to remove all extracellular bacilli, medium was replaced, and incubation was continued. Macrophages were lysed with water-0.05\% Tween 80 to release intracellular bacteria after 4, 24, 72, 120, and $168 \mathrm{~h}$ postinfection. Bacilli were serially 
diluted in PBS-Tween and plated on 7H11 with OADC. Plates were incubated for 3 to 4 weeks for CFU counts. All experiments were performed in triplicate.

Murine infection model and ethics statement. Groups of 6- to 8-week-old BALB/c mice were infected by low-dose aerosol exposure with $M$. tuberculosis H37Rv wild type, $\Delta f 6 / s f d S$, and the complemented strain using a Glas-Col (Terre Haute, IN) aerosol generator calibrated to deliver approximately 100 bacteria into the lungs. Bacterial counts in the lungs $(n=5)$ at each time point of the study were determined by plating serial dilutions of individual lung homogenates on duplicate plates of Middlebrook $7 \mathrm{H} 11$ agar containing OADC enrichment. CFU were counted after 3 to 4 weeks incubation at $37^{\circ} \mathrm{C}$. BALB/C mice were bred and housed under specific pathogen-free conditions at the Medical Research Council, National Institute for Medical Research (NIMR). All mouse studies and breeding were approved by the animal ethics committee at NIMR. Protocols for experiments were performed under project license number 80/2236, in accordance with Home Office (United Kingdom) requirements and the Animal Scientific Procedures Act, 1986.

Data availability. Array design is available in ArrayExpress (accession no. A-BUGS-41). Microarray data have been deposited in ArrayExpress (accession number E-MTAB-9327).

\section{SUPPLEMENTAL MATERIAL}

Supplemental material is available online only.

SUPPLEMENTAL FILE 1, PDF file, 1.2 MB.

SUPPLEMENTAL FILE 2, PDF file, $0.1 \mathrm{MB}$.

\section{ACKNOWLEDGMENTS}

We thank Ruben Hartkoorn and Stewart Cole for the H37Rv $\Delta$ sigF mutant, the Biological Service staff at NIMR, Mill Hill for help with maintaining mice, the $\mathrm{B} \mu \mathrm{G} @ S$ (the Bacterial Microarray Group at St. George's, University of London) for supplying M. tuberculosis microarrays, Finn Werner for critical reading of the manuscript, and Douglas B. Young for support and mentoring.

This work was supported by the British Medical Research Council (MRC; program number U117581288, grant numbers MR/L018519/1 and MR/S009647/1). Funding for open access charge: University College London. The funders had no role in study design, data collection and analysis, decision to publish, or preparation of the manuscript.

We declare no conflicts of interest.

\section{REFERENCES}

1. Arnvig KB, Comas I, Thomson NR, Houghton J, Boshoff $\mathrm{HI}$, Croucher NJ, Rose G, Perkins TT, Parkhill J, Dougan G, Young DB. 2011. Sequence-based analysis uncovers an abundance of non-coding RNA in the total transcriptome of Mycobacterium tuberculosis. PLoS Pathog 7:e1002342. https:// doi.org/10.1371/journal.ppat.1002342.

2. Cortes T, Schubert OT, Rose G, Arnvig KB, Comas I, Aebersold R, Young DB. 2013. Genome-wide mapping of transcriptional start sites defines an extensive leaderless transcriptome in Mycobacterium tuberculosis. Cell Rep 5:1121-1131. https://doi.org/10.1016/j.celrep.2013.10.031.

3. Houghton J, Cortes T, Schubert O, Rose G, Rodgers A, De Ste Croix M, Aebersold R, Young DB, Arnvig KB. 2013. A small RNA encoded in the Rv2660c locus of Mycobacterium tuberculosis is induced during starvation and infection. PLoS One 8:e80047. https://doi.org/10.1371/journal pone.0080047.

4. Miotto P, Forti F, Ambrosi A, Pellin D, Veiga DF, Balazsi G, Gennaro ML, Di Serio C, Ghisotti D, Cirillo DM. 2012. Genome-wide discovery of small RNAs in Mycobacterium tuberculosis. PLoS One 7:e51950. https://doi.org/ 10.1371/journal.pone.0051950.

5. Shell SS, Wang J, Lapierre P, Mir M, Chase MR, Pyle MM, Gawande R, Ahmad R, Sarracino DA, loerger TR, Fortune SM, Derbyshire KM, Wade JT, Gray TA. 2015. Leaderless transcripts and small proteins are common features of the mycobacterial translational landscape. PLoS Genet 11:e1005641. https://doi .org/10.1371/journal.pgen.1005641.

6. Arnvig KB, Young DB. 2009. Identification of small RNAs in Mycobacterium tuberculosis. Mol Microbiol 73:397-408. https://doi.org/10.1111/j.1365-2958 .2009.06777.x.

7. DiChiara JM, Contreras-Martinez LM, Livny J, Smith D, McDonough KA, Belfort M. 2010. Multiple small RNAs identified in Mycobacterium bovis BCG are also expressed in Mycobacterium tuberculosis and Mycobacterium smegmatis. Nucleic Acids Res 38:4067-4078. https://doi.org/10.1093/nar/gkq101.

8. Gerrick ER, Barbier T, Chase MR, Xu R, Francois J, Lin VH, Szucs MJ, Rock JM, Ahmad R, Tjaden B, Livny J, Fortune SM. 2018. Small RNA profiling in
Mycobacterium tuberculosis identifies Mrsl as necessary for an anticipatory iron sparing response. Proc Natl Acad Sci U S A 115:6464-6469. https://doi.org/10.1073/pnas.1718003115.

9. Mai J, Rao C, Watt J, Sun X, Lin C, Zhang L, Liu J. 2019. Mycobacterium tuberculosis 6C sRNA binds multiple mRNA targets via C-rich loops independent of RNA chaperones. Nucleic Acids Res 47:4292-4307. https://doi .org/10.1093/nar/gkz149.

10. Moores A, Riesco AB, Schwenk S, Arnvig KB. 2017. Expression, maturation and turnover of DrrS, an unusually stable, DosR regulated small RNA in Mycobacterium tuberculosis. PLoS One 12:e0174079. https://doi.org/10 .1371 /journal.pone.0174079.

11. Pelly S, Bishai WR, Lamichhane G. 2012. A screen for non-coding RNA in Mycobacterium tuberculosis reveals a cAMP-responsive RNA that is expressed during infection. Gene 500:85-92. https://doi.org/10.1016/j.gene.2012.03.044.

12. Salina EG, Grigorov A, Skvortsova Y, Majorov K, Bychenko O, Ostrik A, Logunova N, Ignatov D, Kaprelyants A, Apt A, Azhikina T. 2019. MTS1338, a small Mycobacterium tuberculosis RNA, regulates transcriptional shifts consistent with bacterial adaptation for entering into dormancy and survival within host macrophages. Front Cell Infect Microbiol 9:405. https:// doi.org/10.3389/fcimb.2019.00405.

13. Solans L, Gonzalo-Asensio J, Sala C, Benjak A, Uplekar S, Rougemont J, Guilhot C, Malaga W, Martin C, Cole ST. 2014. The PhoP-dependent ncRNA Mcr7 modulates the TAT secretion system in Mycobacterium tuberculosis. PLoS Pathog 10:e1004183. https://doi.org/10.1371/journal.ppat.1004183.

14. Lovewell RR, Sassetti CM, VanderVen BC. 2016. Chewing the fat: lipid metabolism and homeostasis during $M$. tuberculosis infection. Curr Opin Microbiol 29:30-36. https://doi.org/10.1016/j.mib.2015.10.002.

15. Hartkoorn RC, Sala C, Uplekar S, Busso P, Rougemont J, Cole ST. 2012. Genome-wide definition of the SigF regulon in Mycobacterium tuberculosis. J Bacteriol 194:2001-2009. https://doi.org/10.1128/JB.06692-11.

16. Betts JC, Lukey PT, Robb LC, McAdam RA, Duncan K. 2002. Evaluation of a nutrient starvation model of Mycobacterium tuberculosis persistence by 
gene and protein expression profiling. Mol Microbiol 43:717-731. https:// doi.org/10.1046/j.1365-2958.2002.02779.x.

17. DeMaio J, Zhang Y, Ko C, Young DB, Bishai WR. 1996. A stationary-phase stress-response sigma factor from Mycobacterium tuberculosis. Proc Natl Acad Sci U S A 93:2790-2794. https://doi.org/10.1073/pnas.93.7.2790.

18. Geiman DE, Kaushal D, Ko C, Tyagi S, Manabe YC, Schroeder BG, Fleischmann RD, Morrison NE, Converse PJ, Chen P, Bishai WR. 2004. Attenuation of late-stage disease in mice infected by the Mycobacterium tuberculosis mutant lacking the SigF alternate sigma factor and identification of SigF-dependent genes by microarray analysis. Infect Immun 72:1733-1745. https://doi.org/10.1128/IAI.72.3.1733-1745.2004.

19. Michele TM, Ko C, Bishai WR. 1999. Exposure to antibiotics induces expression of the Mycobacterium tuberculosis sigF gene: implications for chemotherapy against mycobacterial persistors. Antimicrob Agents Chemother 43:218-225. https://doi.org/10.1128/AAC.43.2.218.

20. Ehlers S, Schaible UE. 2012. The granuloma in tuberculosis: dynamics of a host-pathogen collusion. Front Immunol 3:411. https://doi.org/10.3389/ fimmu.2012.00411.

21. Wayne LG, Hayes LG. 1996. An in vitro model for sequential study of shiftdown of Mycobacterium tuberculosis through two stages of nonreplicating persistence. Infect Immun 64:2062-2069. https://doi.org/10.1128/iai 64.6.2062-2069.1996.

22. Stewart GR, Wernisch L, Stabler R, Mangan JA, Hinds J, Laing KG, Young DB, Butcher PD. 2002. Dissection of the heat-shock response in Mycobacterium tuberculosis using mutants and microarrays. Microbiology (Reading) 148:3129-3138. https://doi.org/10.1099/00221287-148-10-3129.

23. Kery MB, Feldman M, Livny J, Tjaden B. 2014. TargetRNA2: identifying targets of small regulatory RNAs in bacteria. Nucleic Acids Res 42: W124-W129. https://doi.org/10.1093/nar/gku317.

24. Hakiem OR, Parijat P, Tripathi P, Batra JK. 2020. Mechanism of HrcA function in heat shock regulation in Mycobacterium tuberculosis. Biochimie 168:285-296. https://doi.org/10.1016/j.biochi.2019.11.012.

25. Rosser A, Stover C, Pareek M, Mukamolova GV. 2017. Resuscitation-promoting factors are important determinants of the pathophysiology in Mycobacterium tuberculosis infection. Crit Rev Microbiol 43:621-630. https://doi.org/10.1080/1040841X.2017.1283485.

26. Salina EG, Waddell SJ, Hoffmann N, Rosenkrands I, Butcher PD, Kaprelyants AS. 2014. Potassium availability triggers Mycobacterium tuberculosis transition to, and resuscitation from, non-culturable (dormant) states. Open Biol 4. https://doi.org/10.1098/rsob.140106.
27. Shamovsky I, Ivannikov M, Kandel ES, Gershon D, Nudler E. 2006. RNAmediated response to heat shock in mammalian cells. Nature 440:556-560. https://doi.org/10.1038/nature04518.

28. Xue D, Chen Y, Li J, Han J, Liu Y, Jiang S, Zhou Z, Zhang W, Chen M, Lin M, Ongena M, Wang J. 2019. Targeting Hsp20 using the novel small noncoding RNA DnrH regulates heat tolerance in Deinococcus radiodurans. Front Microbiol 10:2354. https://doi.org/10.3389/fmicb.2019.02354.

29. Yura T. 2019. Regulation of the heat shock response in Escherichia coli: history and perspectives. Genes Genet Syst 94:103-108. https://doi.org/ 10.1266/ggs.19-00005.

30. Chen P, Ruiz RE, Li Q, Silver RF, Bishai WR. 2000. Construction and characterization of a Mycobacterium tuberculosis mutant lacking the alternate sigma factor gene, sigF. Infect Immun 68:5575-5580. https://doi.org/10 .1128/IAI.68.10.5575-5580.2000.

31. Orme IM, Basaraba RJ. 2014. The formation of the granuloma in tuberculosis infection. Semin Immunol 26:601-609. https://doi.org/10.1016/j.smim.2014 .09.009.

32. Harper J, Skerry C, Davis SL, Tasneen R, Weir M, Kramnik I, Bishai WR, Pomper MG, Nuermberger EL, Jain SK. 2012. Mouse model of necrotic tuberculosis granulomas develops hypoxic lesions. J Infect Dis 205:595-602 . https://doi.org/10.1093/infdis/jir786.

33. Hinds J, Mahenthiralingam E, Kempsell KE, Duncan K, Stokes RW, Parish T, Stoker NG. 1999. Enhanced gene replacement in mycobacteria. Microbiology (Reading) 145 (Pt 3):519-527. https://doi.org/10.1099/13500872-145 -3-519.

34. Gopaul KK, Brooks PC, Prost JF, Davis EO. 2003. Characterization of the two Mycobacterium tuberculosis recA promoters. J Bacteriol 185:6005-6015. https://doi.org/10.1128/JB.185.20.6005-6015.2003.

35. Springer B, Master S, Sander P, Zahrt T, McFalone M, Song J, Papavinasasundaram KG, Colston MJ, Boettger E, Deretic V. 2001. Silencing of oxidative stress response in Mycobacterium tuberculosis: expression patterns of ahpC in virulent and avirulent strains and effect of ahpC inactivation. Infect Immun 69:5967-5973. https://doi.org/10 .1128/IAI.69.10.5967-5973.2001.

36. Li H, Durbin R. 2009. Fast and accurate short read alignment with Burrows-Wheeler transform. Bioinformatics 25:1754-1760. https://doi.org/10 .1093/bioinformatics/btp324.

37. Li H, Handsaker B, Wysoker A, Fennell T, Ruan J, Homer N, Marth G, Abecasis G, Durbin R, Genome Project Data Processing S. 2009. The Sequence Alignment/Map format and SAMtools. Bioinformatics 25:2078-2079. https://doi .org/10.1093/bioinformatics/btp352. 